\title{
ON CONTINUITY OF DERIVATIONS AND EPIMORPHISMS ON SOME VECTOR-VALUED GROUP ALGEBRAS
}

\author{
RAMEsh V. GaRimella
}

\begin{abstract}
For a locally compact Abelian group $G$ and a commutative Banach algebra $B$, let $L^{1}(G, B)$ be the Banach algebra of all Bochner integrable functions. We show that if $G$ is compact and $B$ is a nonunital Banach algebra without nontrivial zero divisors, then (i) all derivations on $L^{1}(G, B)$ are continuous if and only if all derivations on $B$ are continuous, and (ii) each epimorphism from a Banach algebra $X$ onto $L^{1}(G, B)$ is continuous provided every epimorphism from $X$ onto $B$ is continuous. If $G$ is noncompact then every derivation on $L^{1}(G, B)$ and every epimorphism from a commutative Banach algebra onto $L^{1}(G, B)$ are continuous. Our results extend the results of Neumann and Velasco for nonunital Banach algebras.
\end{abstract}

\section{INTRODUCTION}

In [10] Neumann and Velasco gave some results concerning the automatic continuity of homomorphisms and derivations on $L^{1}(G, B)$, the convolution algebra of all Bochner integrable functions on a locally compact Abelian group $G$ with values in a commutative unital Banach algebra $B$. They showed that if $G$ is a locally compact noncompact Abelian group and $B$ is any commutative unital Banach algebra, then all derivations on $L^{1}(G, B)$ and all epimorphisms from a Fréchet algebra onto $L^{\mathbf{1}}(G, B)$ are continuous. It is also shown that if $G$ is compact, then all derivations on $L^{1}(G, B)$ are continuous precisely when all derivations on $B$ are continuous; and each epimorphism from a Fréchet algebra $X$ onto $L^{1}(G, B)$ is continuous whenever each epimorphism from $X$ onto $B$ is continuous. The proofs given in $[\mathbf{1 0}]$ depend on the fact that $B$ is unital. In this note we extend the above results to a nonunital Banach algebra $B$ under the assumption that $B$ has no nontrivial zero divisors. Our results extend the results of Neumann and Velasco to a larger class of Banach algebras that includes some radical Banach algebras.

\section{Preliminaries}

Let $B$ be a commutative Banach algebra (not necessarily unital), and let $G$ be a locally compact Abelian group with Haar measure $h$ and dual group $\Gamma$. We assume

Received 16th October, 1996.

Copyright Clearance Centre, Inc. Serial-fee code: 0004-9729/97 $\$ A 2.00+0.00$. 
that if $G$ is compact, the Haar measure $h$ is normalised so that $h(G)=1$. Let $L^{1}(G, B)$ denote the Banach algebra of all Bochner integrable functions from $G$ into $B$, and let

$$
(f * g)(s):=\int_{G} f(s-t) g(t) d h(t) \text { for all } f, g \in L^{1}(G, B) \text { and } s \in G
$$

and

$$
\|f\|_{1}:=\int_{G}\|f(t)\| d h(t) \text { for all } f \in L^{1}(G, B) .
$$

It is easy to show that $L^{1}(G, B)$ is also a commutative Banach algebra.

For any $\gamma \in \Gamma$, let $\phi_{\gamma}$ denote the evaluation of the vector-valued Fourier transform at $\gamma$. That is, $\phi_{\gamma}$ is a mapping from $L^{1}(G, B)$ onto $B$ given by

$$
\phi_{\gamma}(f):=\widehat{f}(\gamma):=\int_{G} \overline{\gamma(t)} f(t) d h(t) \text { for all } f \in L^{1}(G, B) .
$$

It can be shown that $\phi_{\gamma}$ is a continuous algebra homomorphism from $L^{1}(G, B)$ onto $B$ for each $\gamma \in \Gamma$. Further, for any $\gamma \in \Gamma$, let

$$
M_{\gamma}:=\left\{f \in L^{1}(G, B): \widehat{f}(\gamma)=\theta\right\}
$$

where $\theta$ is the zero vector of $B$. Clearly $M_{\gamma}$ is a closed ideal of $L^{1}(G, B)$. Notice that if $B$ has no nontrivial zero divisors, then $N_{\gamma}$ is a closed prime ideal of $L^{1}(G, B)$, where an ideal $I$ of a commutative algebra is said to be prime if the product $x y \in I$ only if either $x \in I$ or $y \in I$. It is straightforward to prove that $\bigcap_{\gamma \in \Gamma} M_{\gamma}=\{0\}$. For relevant information on $L^{1}(G, B)$ and for related results in harmonic analysis on Abelian groups we refer to $[\mathbf{5}, \mathbf{8}, \mathbf{1 1}]$.

A closed ideal $\Im$ of a commutative Banach algebra $A$ is said to be a separating ideal of $A$ if, for every sequence $\left\{x_{n}\right\}$ in $A$, there is a positive integer $m$ such that

$$
\overline{x_{1} x_{2} \cdots x_{n} \Im}=\overline{x_{1} x_{2} \cdots x_{m} \Im} \text { for all } n \geqslant m \text {. }
$$

Recall that a linear operator $D: A \rightarrow A$ is called a derivation if $D(x y)=x D y+y D x$ for all $x, y$ in $A$. An algebra homomorphism $h$ from a Banach algebra $B$ to $A$ is called an epimorphism if $h$ is onto. For any linear operator $T$ from a Banach space $X$ into a Banach space $Y$,

$$
\Im(T)=:\left\{y \in Y \mid \exists x_{n} \rightarrow 0 \text { in } X \text { with } T x_{n} \rightarrow y\right\}
$$

is said to be the separating subspace of $T$. It is easy to see that $\Im(T)$ is a closed subspace of $Y$. By the closed graph theorem, $T$ is continuous if and only if $\Im(T)=\{0\}$. For 
any derivation $D$ on a commutative Banach algebra $A$ or any epimorphism $h$ from a Banach algebra $B$ onto $A$, the separating subspace $\Im(D)$ or $\Im(h)$ is a separating ideal of $A$. Finally we note that there can be at most finitely many closed prime ideals not containing a separating ideal $\Im$ of a commutative Banach algebra. For a proof of the above statement we refer to [6].

The following properties of the separating subspace are very useful in some of the proofs of the main results of the paper.

Proposition 2.1. Let $S$ be a linear operator from a Banach space $X$ into a Banach space $Y$, and $R$ be a continuous linear operator from $Y$ into a Banach space $Z$. Then

(i) $R \circ S$ is continuous if and only if $\Im(S)$ is contained in the kernel of $R$,

(ii) $\overline{R(\Im(S))}=\Im(R \circ S)$.

ProOF: See [12].

For relevant information on separating ideals, their relation to the prime ideals of the Banach algebra, and for related results on automatic continuity theory we refer to $[\mathbf{1}, \mathbf{2}, \mathbf{3}, \mathbf{4}, \mathbf{7}, \mathbf{9}, \mathbf{1 2}]$.

For each $\gamma \in \Gamma$ and for any $x \in B$, we let

$$
(\gamma \otimes x)(s):=\gamma(s) x \quad \text { for all } s \in G
$$

If $G$ is a compact group, then $\gamma \otimes x \in L^{1}(G, B)$ and further $\|\gamma \otimes x\|_{1}=\|x\|$. We define $\Phi_{\gamma}$ to be the evaluation of the vector-valued Fourier transform at $\gamma$ in $\Gamma$. We recall some of the properties of the product $\gamma \otimes x$ for future reference. Since the proof of Proposition 2.2 is straightforward, it is left to the reader.

Proposition 2.2. Let $G$ be a compact Abelian group with dual group $\Gamma$, and $B$ be a commutative Banach algebra. Let $x, y \in B, f \in L^{1}(G, B)$, and $\gamma$ be a nontrivial character in $\Gamma$. Then

(i) $\Phi_{\gamma}(\gamma \otimes x)=x$;

(ii) $\gamma \otimes(\alpha x+\beta y)=\alpha(\gamma \otimes x)+\beta(\gamma \otimes y)$ for all scalars $\alpha$ and $\beta$;

(iii) $(\gamma \otimes x y)=(\gamma \otimes x) *(\gamma \otimes y)$;

(iv) $(\gamma \otimes x \widehat{f}(\gamma))=(\gamma \otimes x) *(\gamma \otimes \widehat{f}(\gamma))=(\gamma \otimes x) * f$;

(v) If $B$ has multiplicative identity 1 , then $\gamma \otimes 1$ is an idempotent in $L^{1}(G, B)$, and further $(\gamma \otimes \widehat{f}(\gamma))=(\gamma \otimes 1) * f$.

\section{Main Results}

The following lemma is the key to the continuity of derivations on $L^{1}(G, B)$. 
LEMMA 3.1. Let $G$ be a compact Abelian group, $B$ be a commutative Banach algebra without nontrivial zero divisors, and $\gamma$ be a character in $\Gamma$. Then

(i) for any derivation $\delta$ on $L^{1}(G, B)$, there exists a unique derivation $D=$ $D(\gamma, \delta)$ on $B$ such that the following diagram commutes:

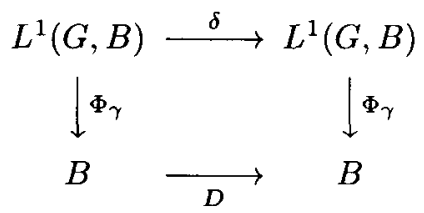

(ii) for any derivation $D$ on $B$, and for a given nonzero element $z$ in $B$, there exists a derivation $\delta=\delta(D, \gamma, z)$ on $L^{1}(G, B)$ such that the following diagram commutes:

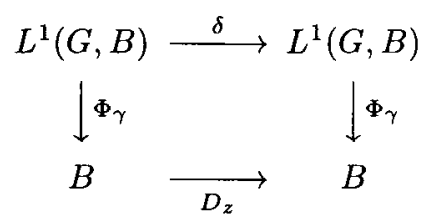

where $D_{z}$ is the derivation defined by $D_{z}(x)=z D(x)$ for all $x$ in $B$.

Proof: (i) Let $\delta$ be a derivation on $L^{1}(G, B)$ and $\gamma \in \Gamma$. Define $D: B \rightarrow B$ by $D(x)=\Phi_{\gamma}(\delta(\gamma \otimes x))$ for all $x \in B$. By using the properties (i) and (ii) of Proposition 2.2 , it is easy to show that $D$ is a derivation on $B$. Now we show that the diagram commutes. Fix a nonzero element $w$ in $B$. For any $f \in L^{1}(G, B)$, we have

$$
\begin{aligned}
D(w \widehat{f}(\gamma)) & =\Phi_{\gamma}(\delta(\gamma \otimes w \widehat{f}(\gamma))) \\
& =\Phi_{\gamma}(\delta((\gamma \otimes w) * f)) \quad(\text { by (iv) of Proposition 2.2) } \\
& =\Phi_{\gamma}[(\gamma \otimes w) * \delta f+\delta(\gamma \otimes w) * f]
\end{aligned}
$$

and so

$$
D(w \widehat{f}(\gamma))=w \Phi_{\gamma}(\delta f)+\Phi_{\gamma}(\delta(\gamma \otimes w)) \widehat{f}(\gamma)
$$

On the other hand,

$$
D(w \widehat{f}(\gamma))=w D(\widehat{f}(\gamma))+D(w) \widehat{f}(\gamma)
$$

Now the above equations (1) and (2) imply that

$$
w\left[D(\widehat{f}(\gamma))-\Phi_{\gamma}(\delta f)\right]=\theta
$$


Since $w$ is a nonzero vector, and $B$ has no nontrivial zero divisors, $D(\widehat{f}(\gamma))=\Phi_{\gamma}(\delta f)$, as required.

If $D_{1}$ is any derivation on $B$ such that the diagram is commutative, then for any $x$ in $B$ we have

$$
\begin{aligned}
D_{1}(x) & =D_{1}\left(\Phi_{\gamma}(\gamma \otimes x)\right) \quad \text { (by (i) of Proposition 2.2) } \\
& =\Phi_{\gamma}(\delta(\gamma \otimes x))=D\left(\Phi_{\gamma}(\gamma \otimes x)\right)=D(x) .
\end{aligned}
$$

Thus $D$ is unique.

(ii) Let $D$ be a derivation on $B$ and $z$ be a nonzero element in $B$. Define the mapping $\delta: L^{1}(G, B) \rightarrow L^{1}(G, B)$ by $\delta(f)=\gamma \otimes z D(\widehat{f}(\gamma))$. Then it is easy to show that $\delta$ is a derivation on $L^{1}(G, B)$. The rest of the proof is similar to the proof in part (i).

Now we are ready for one of the main results.

Theorem 3.2. Let $G$ be a compact Abelian group and $B$ be a commutative $B$ anach algebra without nontrivial zero divisors. Then

(i) all derivations on $L^{1}(G, B)$ are continuous if and only if all derivations on $B$ are continuous;

(ii) every epimorphism from a Banach algebra $X$ onto $L^{1}(G, B)$ is continuous provided every epimorphism from $X$ onto $B$ is continuous.

Proof: (i) We first suppose that all derivations on $B$ are continuous. Let $\delta$ be any derivation on $L^{1}(G, B)$, and $\gamma$ be a continuous character on $G$. By Lemma 3.1(i), there exists a derivation $D=D(\gamma, \delta)$ on $B$ such that

$$
D \circ \Phi_{\gamma}=\Phi_{\gamma} \circ \delta
$$

By assumption $D$ is continuous, and so $\Phi_{\gamma} \circ \delta$ is continuous. Therefore by (i) of Proposition 2.1, the separating ideal $\Im(\delta)$ of $\delta$ is contained in $M_{\gamma}$. Since $\gamma$ is an arbitrary continuous character and $\bigcap_{\gamma \in \Gamma} M_{\gamma}=\{0\}$, it follows that $\Im(\delta)=\{0\}$. Hence $\delta$ is continuous.

Conversely, suppose that all derivations on $L^{1}(G, B)$ are continuous. Let $D$ be a derivation on $B$ and $z$ be a fixed nonzero element in $B$. By Lemma 3.1(ii), for each $\gamma \in \Gamma$, there exists a derivation $\delta$ on $L^{1}(G, B)$ such that $\Phi_{\gamma} \circ \delta=D_{z} \circ \Phi_{\gamma}$. We claim $D_{z}$ is continuous. Let $x_{n} \rightarrow \theta$ in $B$, and suppose $D_{z}\left(x_{n}\right) \rightarrow y$ in $B$. Since $\left\|\gamma \otimes x_{n}\right\|_{1}=\left\|x_{n}\right\|, \quad \gamma \otimes x_{n} \rightarrow 0$ in $L^{1}(G, B)$. Because $\delta$ is continuous and $\Phi_{\gamma} \circ \delta=D_{z} \circ \Phi_{\gamma}$, it follows that $\left(D_{z} \circ \Phi_{\gamma}\right)\left(\gamma \otimes x_{n}\right) \rightarrow \theta$. However, $\Phi_{\gamma}\left(\gamma \otimes x_{n}\right)=x_{n}$. Hence $D_{z}\left(x_{n}\right) \rightarrow \theta$. Therefore $y=\theta$. This proves that $D_{z}$ is continuous, and hence 
$\Im\left(D_{z}\right)=\{\theta\}$. By Proposition 2.1(ii), $\overline{z \Im(D)}=\Im\left(D_{z}\right)=\{\theta\}$. Since $B$ has no nontrivial zero divisors, and $z$ is a nonzero element in $B, \Im(D)=\{\theta\}$. Hence $D$ is continuous.

(ii) Suppose that every epimorphism from a Banach algebra $X$ onto $B$ is continuous. Let $h: X \rightarrow L^{1}(G, B)$ be an epimorphism and fix $\gamma$ in $\Gamma$. Since $\Phi_{\gamma}: L^{\mathbf{1}}(G, B) \rightarrow B$ is an epimorphism, $\Phi_{\gamma} \circ h: X \rightarrow B$ is also an epimorphism. By our assumption, $\Phi_{\gamma} \circ h$ is continuous and hence $\Im(h)$ is contained in $M_{\gamma}$. Since $\gamma$ is arbitrary and $\bigcap_{\gamma \in \Gamma} M_{\gamma}=\{0\}$, it follows that $h$ is continuous.

Now we turn to the case where $G$ is a noncompact locally compact Abelian group.

THEOREM 3.3. Let $G$ be a noncompact locally compact Abelian group, and $B$ be a commutative Banach algebra without nontrivial zero divisors. Then every derivation on $L^{1}(G, B)$ is continuous. Also, every epimorphism from a commutative Banach algebra onto $L^{1}(G, B)$ is continuous.

Proof: First we show that every derivation on $L^{1}(G, B)$ is continuous. Let $\delta$ be a derivation on $L^{1}(G, B)$. To prove that $\delta$ is continuous, it suffices to show that $\Im(\delta)=\{0\}$. Since $\bigcap_{\gamma \in \Gamma} M_{\gamma}=\{0\}$, it is enough to show $\Im(\delta)$ is contained in $M_{\gamma}$ for each $\gamma \in \Gamma$. Since any separating ideal of an algebra is contained in all but finitely many closed prime ideals of the algebra, and since each $M_{\gamma}$ is a closed prime ideal in $L^{1}(G, B)$, there exists at most finitely many continuous characters $\gamma_{1}, \gamma_{2}, \ldots, \gamma_{n}$ such that $\Im(\delta)$ is not contained in $M_{\gamma_{i}}$ for $i=1,2, \ldots, n$. Let $f \in\left(\Im(\delta) \cap\left(\bigcap_{i=2}^{n} M_{\gamma_{i}}\right)\right) \backslash M_{\gamma_{1}}$. Since $M_{\gamma_{1}}$ is a prime ideal, such a function $f$ exists. By the Hahn-Banach theorem, since $\widehat{f}\left(\gamma_{1}\right) \neq \theta$, there exists a continuous linear functional $\lambda$ on $B$ such that $\lambda\left(\widehat{f}\left(\gamma_{1}\right)\right) \neq 0$. Now consider the basic open set

$$
N:=\left\{\gamma \in \Gamma:\left|\lambda(\widehat{f}(\gamma))-\lambda\left(\widehat{f}\left(\gamma_{1}\right)\right)\right|<\left|\lambda\left(\widehat{f}\left(\gamma_{1}\right)\right)\right|\right\}
$$

of $\Gamma$ containing $\gamma_{1}$. Since $G$ is a noncompact Abelian group, the dual group $\Gamma$ is not discrete. Hence $\gamma_{1}$ is not isolated in $\Gamma$. Obviously, the choice of $f$ implies that the characters $\gamma_{2}, \gamma_{3}, \cdots, \gamma_{n}$ do not belong to $N$. Hence there is a character $\gamma_{0} \in$ $\Gamma-\left\{\gamma_{1}, \gamma_{2}, \cdots, \gamma_{n}\right\}$ such that $\gamma_{0} \in N$. Since $\Im(\delta)$ is contained in $M_{\gamma_{0}}, \widehat{f}\left(\gamma_{0}\right)=\theta$. Hence

$$
\left|\lambda\left(\widehat{f}\left(\gamma_{1}\right)\right)\right|=\left|\lambda\left(\widehat{f}\left(\gamma_{1}\right)\right)-\lambda(\widehat{f}(\gamma))\right|<\left|\lambda\left(\widehat{f}\left(\gamma_{1}\right)\right)\right|
$$

This is a contradiction and so $\Im(\delta) \subseteq M_{\gamma}$ for all $\gamma \in \Gamma$, and $\delta$ must be a continuous derivation.

A similar argument shows that every epimorphism from a Banach algebra onto $L^{1}(G, B)$ is continuous. 
REMARK. Even though in the statement (ii) of Theorem 3.2 we assumed that $X$ is a commutative Banach algebra, the proof can be modified if $X$ is a Fréchet algebra. Similarly in Theorem 3.3, it can be shown that every epimorphism from a Fréchet algebra onto $L^{\mathbf{1}}(G, B)$ is continuous.

\section{REFERENCES}

[1] W.G. Bade and P.C. Curtis, Jr., J. Funct. Anal. 29 (1978), 88-103.

[2] P.C. Curtis, Jr., 'Derivations on commutative Banach algebras', in Proceedings, Long Beach, 1981, Lecture Notes in Math. 975 (Springer-Verlag, Berlin, Heidelberg, New York, 1983), pp. 328-333.

[3] J. Cusack, 'Automatic continuity and topologically simple radical Banach algebras', $J$. London Math. Soc. 16 (1977), 493-500.

[4] H.G. Dales, 'Automatic continuity: A survey', Bull. London Math. Soc. 10 (1978), $129-183$.

[5] J. Diestel and J.J. Uhl, Vector measures, Math. Surveys 15 (Amer. Math. Soc,, Providence, RI, 1977).

[6] R. Garimella, 'On separating ideals of commutative Banach algebras', in Lecture Notes in Pure and Applied Mathematics 175 (Marcel Dekker, Inc., 1996), pp. 181-185.

[7] R. Garimella, 'On nilpotency of the separating ideal of a derivation', Proc. Amer. Math. Soc. 117 (1993), 167-174.

[8] G.P. Johnson, 'Space of function with values in a Banach algebra', Trans. Amer. Math. Soc. 92 (1959), 411-429.

[9] M.M. Neumann, 'Automatic continuity of linear operators', in Functional analysis, surveys and recent results $I I \mathbf{3 8}$, North-Holland Math. Studies (North-Holland, Amsterdam, Ney York, 1980), pp. 269-296.

10] M.M Neumann and M.V. Velasco, 'Continuity of epimorphisms and derivations on vector-valued group algebras', (preprint).

11] W. Rudin, Fourier analysis on groups (Interscience, New York, London, 1962).

12] A.M. Sinclair, Automatic continuity of linear operators, London Math. Soc. Lecture Notes 21 (Cambridge University Press, Cambridge, 1976).

Deparment of Mathematics

Tennessee Technological University

Cookeville TN 38505

United States of America

e-mail: RVG0037@tntech.edu 